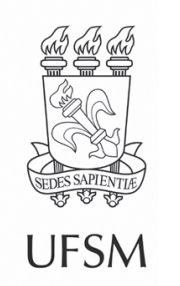

\title{
Artigos
}

\section{Avaliação de incêndio em ambiente de Caatinga a partir de imagens Landsat-8, índice de vegetação realçado e análise por componentes principais}

\author{
Fire analysis in the Caatinga environment from Landsat-8 images, \\ enhanced vegetation index and analysis by the main components
}

Juarez Antônio da Silva Junior ${ }^{1}{ }^{\circ}$, Admilson da Penha Pacheco ${ }^{\circ}$

' Universidade Federal de Pernambuco, Recife, PE, Brasil

\section{RESUMO}

O fogo é um fator importante na perturbação e perda de florestas secas tropicais globais. Os incêndios florestais exercem um papel ecológico relevante, pois afetam a biodiversidade local, as propriedades do solo e o suprimento de água. O bioma Caatinga apresenta um alto nível de degradação de atividades antrópicas e naturais, sendo extremamente afetado por incêndios originados predominantemente por atividades humanas. O sensoriamento remoto orbital, por apresentar características espaciais, espectrais e temporais específicas, é uma alternativa tecnológica imprescindível no monitoramento de áreas afetadas pelo fogo na superfície terrestre. Este trabalho teve como objetivo analisar, no âmbito espacial, espectral e temporal, o comportamento de um incêndio em ambiente de Caatinga a partir de Imagens Landsat-8, Índice de Vegetação Realçado e Análise por Componentes Principais. A quantificação de características da vegetação derivada do índice espectral fornece uma melhor avaliação da condição física da superfície terrestre sob efeitos do fogo. Técnicas de sensoriamento remoto e estatística multivariada foram utilizadas para avaliar comportamento espectral da vegetação nativa exposta a eventos de incêndio do bioma Caatinga. Os resultados do Teste de Normalidade Kolmogorov-Smirnov apresentaram um nível de significância de 5\%. A integração dos métodos estatísticos de Regressão Linear Simples e Análise por Componentes Principais possibilitaram diagnósticos importantes nas estimativas e/ou relacionamentos entre as variáveis aleatórias. A técnica multivariada permitiu avaliar $94 \%$ da variação de dados. Os mapas resultantes da metodologia testada representam um aprimoramento importante no mapeamento da distribuição da vegetação. Este estudo gera indicativos para futuras pesquisas científicas vinculadas ao gerenciamento do espaço relacionado à vulnerabilidade e recuperação de paisagens de vegetação do clima semiárido sob situações de fogo geradas por incêndios.

Palavras-chave: Cobertura Vegetal; Incêndios; Sensoriamento Remoto e Estatística 


\section{ABSTRACT}

Fires generate negative environmental and socioeconomic impacts that directly and indirectly influence the Earth's regional and global climate changes. Forest fires and fires play a relevant ecological role as they affect the local biodiversity, soil properties and water supply. The Caatinga biome has a high level of degradation of human and natural activities, being extremely affected by fires that burn predominantly due to human activities. Remote orbital sensing, as it presents specific spatial, spectral and temporal characteristics, is an essential technological alternative in monitoring areas affected by fire on the Earth's surface. This work aimed to analyze, in a spatial, spectral and temporal scope, the behavior of a fire in a Caatinga environment from the multivariate statistical analysis of Landsat-8 Images data, Enhanced Vegetation Index and Analysis by the Major Components. The quantification of characteristics of vegetation derived from the spectral index provides a better assessment of the physical condition of the earth's surface under the effects of fire. Remote sensing techniques and multivariate statistics were used to assess the spectral behavior of wildfires in the Caatinga biome. The results of the KolmogorovSmirnov Normality Test showed a significance level of 5\%. The integration of the statistical methods of Simple Linear Regression and Analysis by the Principal Components enabled important diagnoses in the estimates and/or relationships between the random variables. The multivariate technique allowed $94 \%$ of the data variation to be assessed. The maps resulting from the tested methodology represent an important improvement in mapping the distribution of vegetation. This study generates indications for future scientific research related to the management of space concerning to vulnerability and recovery of vegetation landscapes from the semi-arid climate under fire situations generated by burnings.

Keywords: Vegetation Cover; Fires; Remote Sensing and Statistics

\section{INTRODUÇÃO}

Conforme Bowman et al. (2009), o fogo é um fenômeno mundial que aparece no registro geológico logo após o surgimento de plantas terrestre, influenciando os padrões e processos globais dos ecossistemas, envolvendo a distribuição e estrutura da vegetação, o ciclo do carbono e o clima. Embora os seres humanos e o fogo sempre coexistam, a capacidade de gerenciar o fogo permanece imperfeita e pode se tornar mais difícil no futuro, à medida que as mudanças climáticas alterarem os regimes de incêndio.

As condições meteorológicas são fatores que influenciam diretamente as etapas do incêndio. Os incêndios apresentam impactos significativos em nível microclimatológico, ambiental e socioeconômico. De acordo com Fernandes et al. (2016), a área incendiada é controlada por uma grande variedade de fatores estáticos 
e dinâmicos, entre eles a cobertura do solo (vegetação), variáveis meteorológicas e as atividades antrópicas, incluindo o desenvolvimento do solo, densidade de estradas, distância de assentamentos, prevenção de incêndios e estratégias contrastantes. Conforme o IPCC (INTERGOVERNMENTAL PANEL ON CLIMATE CHANGE, 2013), Painel Intergovernamental sobre Mudanças Climáticas, as mudanças climáticas tendem a aumentar os riscos de grandes incêndios na Terra.

A Caatinga apresenta um alto nível de degradação por atividades antrópicas e fenômenos naturais, relacionados com aspectos climáticos, tais como a seca, altas temperaturas, deficit de água, baixa umidade e ocorrência de incêndios (MARIANO et al., 2018). O bioma Caatinga é extremamente afetado pelos incêndios originados, predominantemente, por atividades humanas.

Imagens de diferentes satélites de sensoriamento remoto têm sido utilizadas para gerenciar as fases da dinâmica de um incêndio, como o objetivo de estimar as condições de perigo, detectar o comportamento do fogo e analisar os efeitos da recuperação da vegetação (LIZUNDIA-LOIOLA et al.,2020). Estudos recentes de sensoriamento remoto aplicado no monitoramento do bioma Caatinga são apresentados nos trabalhos de Campos et al. (2019).

Os índices de vegetação são ferramentas de sensoriamento remoto, que visam caracterizar as propriedades espectrais e parâmetros biofísicos da vegetação a partir da razão de diferenças de bandas espectrais das imagens, indicando a quantidade e a qualidade da vegetação observada em termos de biomassa, área foliar, vigor vegetativo, teor de clorofila e atividade fotossintética (HUETE et al., 1997; PONZONI; SHIMABUKURO, 2010; THENKABAIL; LYON; HUETE, 2016).

Dentre os inúmeros índices de vegetação existentes, destaca-se o índice EVI (Enhanced Vegetation Index), proposto por Huete et al. (1997), que minimiza os problemas relacionados à saturação, interferência do solo e da atmosfera na resposta da vegetação, apresentando assim mais complexidade do que o NDVI (Normalized Difference Vegetation Index) (HUETE et al., 1997; THENKABAIL; LYON; HUETE, 2016). O 
EVI é mais sensível às variações na resposta estrutural do dossel, incluindo o índice de área foliar, a fisionomia da planta e a arquitetura do dossel (GAO et al., 2000; HUETE et al., 2006). Estudos realizados com imagens de diferentes satélites têm demostrado o potencial do EVI na análise de padrões temporais de recuperação da vegetação (WALLACE; WEBB; THOMAS, 2008; GALVÃO et al., 2011).

A análise digital de imagens de sensoriamento remoto permite a modelagem estatística de dados a partir de métodos simples e multivariados, tais como técnicas de regressão linear e análise por componentes principais. Os módulos matemáticos e estatísticos aplicados em dados de sensoriamento remoto permitem a realização de testes de hipóteses, estimativas e análises das tendências (espaciais, espectrais e temporais) associadas à resposta fisiológica da vegetação.

Nesse contexto, este trabalho teve como objetivo analisar, no âmbito espacial, espectral e temporal, o comportamento de incêndios em ambiente de Caatinga como objetivo analisar, no âmbito espacial, espectral e temporal, o comportamento de um incêndio em ambiente de Caatinga a partir de Imagens Landsat-8, Índice de Vegetação Realçado e Análise por Componentes Principais. O evento de incêndio aconteceu em 04/10/2019 no município de Floresta, Pernambuco, próximo ao reservatório de Itaparica, que faz parte da Usina Hidrelétrica Luiz Gonzaga da CHESF (Companhia Hidroelétrica do São Francisco).

\section{MATERIAIS E MÉTODOS}

\section{1 Área de Estudo}

Aárea de estudo compreende um polígono delimitado por coordenadas geográficas

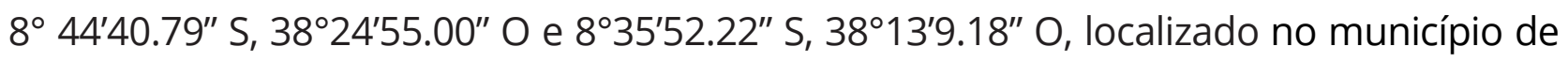
Floresta, semiárido pernambucano. O limite da área de estudo localiza-se próximo ao Reservatório de Itaparica, que integra o complexo hidroelétrico de Paulo Afonso e tem a importância de abastecer os municípios próximos e favorecer a irrigação agrícola. 
De acordo com a classificação da literatura, o clima da região é semiárido de estepes (BShw') quente e seco, apresentando chuvas médias anuais de 410 a 610 mm e duas estações sazonais definidas. As temperaturas médias anuais apresentamse de 24 a $26^{\circ} \mathrm{C}$, com a evaporação na ordem de $3.000 \mathrm{~mm}$ anuais. A vegetação é basicamente composta por Caatinga Hiperxerófila com trechos de Floresta Caducifólia (COMPANHIA DE PESQUISA DE RECURSOS MINERAIS, 2005).

Figura 1 - Localização da área e estudo

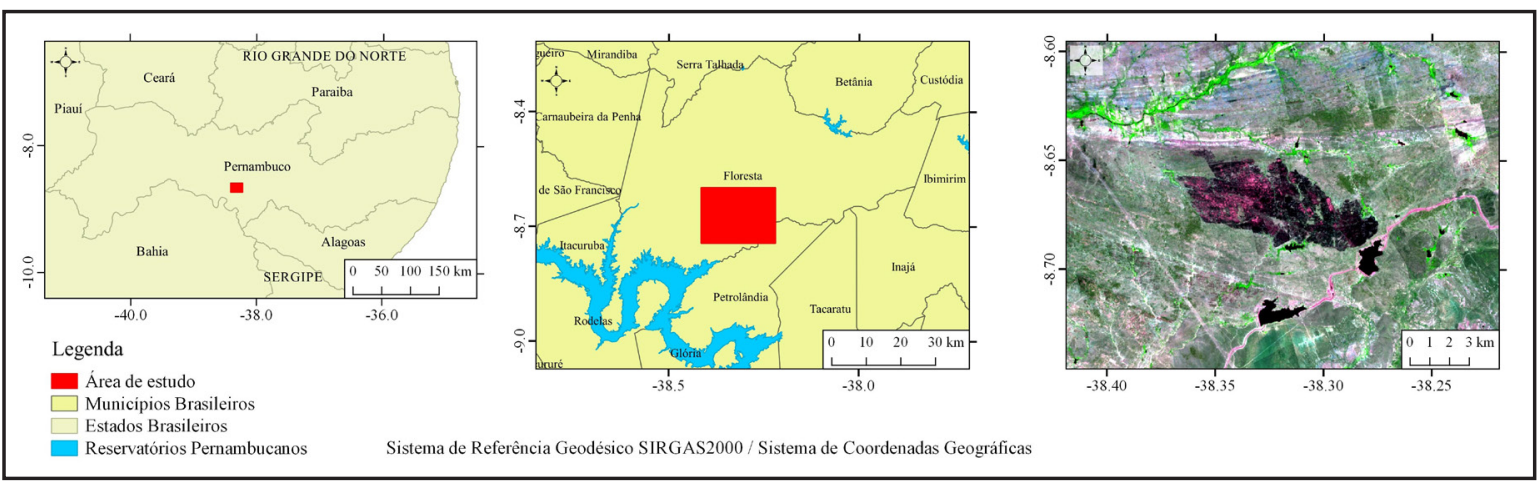

Fonte: IBGE (2016), United States Geological Survey (2019)

\subsection{Descrição do Evento de Incêndio no município de Floresta-PE}

Um incêndio florestal é um fogo que se propaga sem controle, em uma área florestal, afetando os combustíveis vegetais, flora e fauna. Um incêndio florestal distingue-se de outros tipos de incêndio por sua ampla extensão, a velocidade com a qual se pode estender desde o seu lugar de origem, o seu potencial para mudar de direção inesperadamente, e a sua capacidade para superar obstáculos como estradas e rios (MOLINA, 1997). De acordo com informações dos órgãos de segurança pública do município, o incêndio no município de Floresta iniciou-se no dia 04/11/2019 e se alastrou por aproximadamente $15 \mathrm{~km}^{2}$, devastando a vegetação nativa da região até o dia 08/11/2019.0 avanço das chamas foi devido ao calor intenso, além de fortes ventos e da vegetação seca, característicos da Caatinga. 


\subsection{Aquisição e Processamento de Dados}

As imagens utilizadas foram cenas do satélite Landsat-8/OLI relativas às bandas 2 (azul) $(0,45-0,51 \mu \mathrm{m}), 4$ (vermelho) $(0,64-0,67 \mu \mathrm{m})$ e 5 (infravermelho próximo) $(0,85$ - 0,88 $\mu \mathrm{m}$ ), datadas de 15 de outubro de 2019, 16 de outubro de 2019 e 02 de dezembro de 2019. Períodos anteriores e posteriores ao incêndio (04/10/2019) e a regeneração da área, respectivamente. As imagens Landsat 8, corrigidas radiometricamente (produto Nível 1T), foram baixadas do USGS Global Visualization Viewer (2019) e convertidas em reflectância do topo da atmosfera usando os coeficientes de redimensionamento fornecidos nos arquivos de metadados. Esses dados foram georreferenciados para o sistema de referência geodésico SIRGAS2000 na projeção cartográfica UTM fuso 24S. Nas etapas de pré e pós-processamento das imagens utilizou-se o software QGIS 2.14.18. As análises estatísticas foram realizadas na plataforma RStudio 3.6.2. Os softwares utilizados neste estudo são gratuitos e de acesso livre.

A correção atmosférica foi obtida por meio do método empírico DOS (Dark Object Subtraction) desenvolvido por Chavez Junior (1988), denominado como correção atmosférica pelo objeto escuro. De acordo com o mesmo autor, a correção é aplicada nos dados de reflectância no topo da atmosfera através da seleção dos valores no histograma e posteriormente subtraídos de cada banda. A absorção atmosférica não entra na modelagem e a interferência atmosférica é estimada diretamente a partir dos níveis de cinza da imagem Landsat.

Para compreensão dos fenômenos em estudo, aplicou-se o índice espectral EVI proposto por Huete et al. (1997) - Equação (1).

$$
E V I=\frac{\mathrm{G}\left(\rho_{\text {nir }}-\rho_{\text {red }}\right)}{\left(\mathrm{L}+\mathrm{C}_{1} \rho_{\text {red }}+\mathrm{C}_{2} \rho_{\text {blue }}+\rho_{\text {nir }}\right)}
$$

Em que: $\rho_{\text {nir }}=(0,851-0,879 \mu \mathrm{m}) ; \rho_{\text {red }}=(0,636-0,673 \mu \mathrm{m}) ; \rho_{\text {blue }}=(0,452-0,512 \mu \mathrm{m}) ; \mathrm{L}=$ fator de ajuste para o solo; $\mathrm{G}=$ fator de ganho; $\mathrm{C} 1$ e $\mathrm{C} 2$ = coeficientes de ajuste para efeito de aerossóis na atmosfera. Os valores dos coeficientes utilizados na equação para obtenção do EVI foram: $L=1, C 1=6, C 2=7,5$ e G = 2,5 (HUETE et al., 1997). 
A análise exploratória dos dados é usada para comparações e predições para populações a partir de dados amostrais, uma vez que geralmente os dados de sensoriamento remoto não são viáveis para realizar análise de todos os membros de uma população, que muitas vezes se apresentam por superfícies terrestres de geometria complexa. O teste de normalidade vem como uma hipótese eficiente de diagnóstico de distribuição amostral, pois é formulada observando-se a população testada na amostra. Esse tipo de verificação é necessário, pois se o tamanho da amostra for muito pequeno, as informações podem ser negligenciadas e se o tamanho amostral for muito grande, ocorrerá um desperdício de recursos e tempo (MISHRA et al., 2019; ABOELGHAR et al., 2010).

Estabeleceu-se para a aplicação do índice EVI, o número de 100 amostras de pixels dentro do polígono afetado pelo incêndio. Essas amostras foram coletadas aleatoriamente.

O teste de normalidade tem como objetivo verificar o comportamento de distribuição normal dos dados e, com isso, validar o uso de estatística paramétrica para a análise do conjunto amostral. Neste artigo, um número adequado de pixels/ amostras foi calculado pelo Teste de Normalidade de Kolmogorov-Smirnov antes da execução da pesquisa.

Os testes de normalidade são baseados em características diferentes, como distribuição empírica, momentos, correlação e regressão, e baseados em propriedades especiais da distribuição de dados (ISLAM, 2019). Utilizou-se neste estudo o algoritmo teste de Kolmogorov-Smirnov, apresentado na Equação (2). Dado n valores, ordenados, $x_{1}<x_{2}<\ldots x_{n^{\prime}}$

$\mathrm{KS}=\operatorname{supx}\left|F^{*}(\mathrm{x})-\mathrm{Fn}(\mathrm{x})\right|$

Em que: $F^{*}(x)$ é a função de distribuição hipotética; $F n(x)$ é a função de distribuição empírica estimada com base na amostra aleatória. No geral, a análise baseia-se na hipótese de se o valor de Dn estiver acima do valor de referência descrito em KolmogorovSmirnov e o valor p acima do nível de significância $\sigma$ aceita-se a hipótese nula, na qual os dados seguem uma distribuição normal (CORDER; FOREMAN, 2011). 
Uma "Regressão Linear Simples" foi realizada para determinar a relação entre os dois conjuntos de imagens. A Equação (3) apresenta a modelagem explicativapreditiva dos dados extraídos pelo índice de vegetação (EVI):

$$
Y=a+b X
$$

Em que: $X$ é a variável explicativa (contagem do lago pantanal); $Y$ é a variável dependente; $b$ é $a$ inclinação da reta; $a$ é o intercepto em $Y$. Para sua validação foram utilizados o teste de hipótese através do nível de significância testado pelo valor $p$ e os coeficientes de determinação R-quadrado $\left(\mathrm{R}^{2}\right)$.

Paralelamente, utilizou-se a técnica estatística multivariada de "Análises por Componentes Principais" (ACP). O método ACP é uma transformação ortogonal linear, que transforma o conjunto de dados original em um conjunto de dados compactado de variáveis não correlacionadas, conhecidas como componentes principais (CPS). As CPs representam as informações importantes do conjunto de dados primários (DENG et al., 2019). Para modelar as variações de cada parâmetro específico, em um determinado intervalo de tempo, utilizou-se o modelo ACP proposto por Gaitani et al. (2017). Este modelo foi aplicado em valores específicos temporalmente na escala amostral de pixels, conforme as Equações (4), (5), (6) e (7) abaixo:

$$
\left(\begin{array}{ccc}
x_{1,1} & \cdots & x_{1, n} \\
\vdots & \ddots & \vdots \\
x_{6,1} & \cdots & x_{6, n}
\end{array}\right)
$$

Em que: $n$ representa número de pixels; $x$ o número de bandas.

Considerando cada banda como um vetor, a matriz acima pode ser simplificada, mostrada na Equação (5):

$$
x_{k}=\left(\begin{array}{c}
x_{1} \\
\vdots \\
x_{6}
\end{array}\right)
$$

Em que: $x_{k}$ é o número de bandas.

Para reduzir a dimensionalidade das bandas originais, os valores próprios da 
matriz de covariância devem ser calculados. Essa matriz pode ser calculada da seguinte maneira, conforme Equação (6):

$$
C_{b, b}=\left(\begin{array}{ccc}
\sigma_{1,1} & \cdots & \sigma_{1,6} \\
\vdots & \ddots & \vdots \\
\sigma_{6,1} & \cdots & \sigma_{6,6}
\end{array}\right)
$$

Em que: $i, j$ é a covariância de cada par de bandas diferentes.

$$
\sigma_{1, j}=\frac{1}{N-1} \sum_{p=1}^{N}\left(D N_{p, i} \mu_{i}\right)\left(D N_{p, i} \mu_{j}\right)
$$

Em que: $D N_{p, i}$ é um número digital de um pixel $p$ na banda $i ; j$ é um número digital de um pixel $p$ na banda $j ; \mu_{i}$ e $\mu_{j}$ são as médias do $D N$ para as bandas $i$ e $j$, respectivamente.

A partir da matriz de variância-covariância, os valores próprios são calculados como as raízes da equação característica, na Equação (8):

$$
\operatorname{det}(C-\lambda I)=0
$$

Em que: $C$ é a matriz de covariância das bandas e $I$ é a matriz de identidade diagonal. Os valores próprios indicam as informações originais que eles retêm.

Desses valores, a porcentagem da variação original explicada por cada componente principal pode ser obtida calculando-se a razão de cada autovalor em relação à soma de todos eles (CHUVIECO, 2010). Aqueles componentes que contêm variação mínima e, portanto, as informações mínimas podem ser descartadas. A saída CP1 pode conter valores negativos e positivos. Um valor mais alto e positivo de um pixel na CP1 indica que os valores desse pixel foram grandes e inalterados ao longo do tempo. Por outro lado, um valor mais baixo e mais negativo de um pixel na CP1 indica que os valores desse pixel foram baixos e inalterados ao longo do tempo.

Técnicas estatísticas de regressão linear e análise por componentes principais foram utilizadas por etapas para criar dois modelos de cobertura afetada por incêndio e o processo de regeneração em relação à superfície não incendiada com dados gerados de índice de vegetação EVI. Nos dois modelos, os dados dependentes foram 
a variável EVIpré-fogo e o EVIreg. Para tanto, foi utilizado o método de interceptação de estatística multivariada e validação das amostras pelo algoritmo de KolmogorovSmirnov e através do valor-p da regressão. Os mapas estão representados na escala de 1:175.400, compatível com a tolerância de acuidade visual e qualidade instrumental de produtos cartográficos obtidos por dados raster de precisão espacial de 30 metros.

\section{RESULTADOS E DISCUSSÃO}

A partir da álgebra de bandas das imagens do satélite Landsat-8/OLI foi possível a geração de mapas de distribuição de pixels e índices de vegetação EVI associados, conforme representado na Figura 2.

Figura 2 - Distribuição de pixels de EVl: a) Pré-fogo; b) Pós-fogo; c) Regeneração e d) Distribuição e histograma das amostras em análise

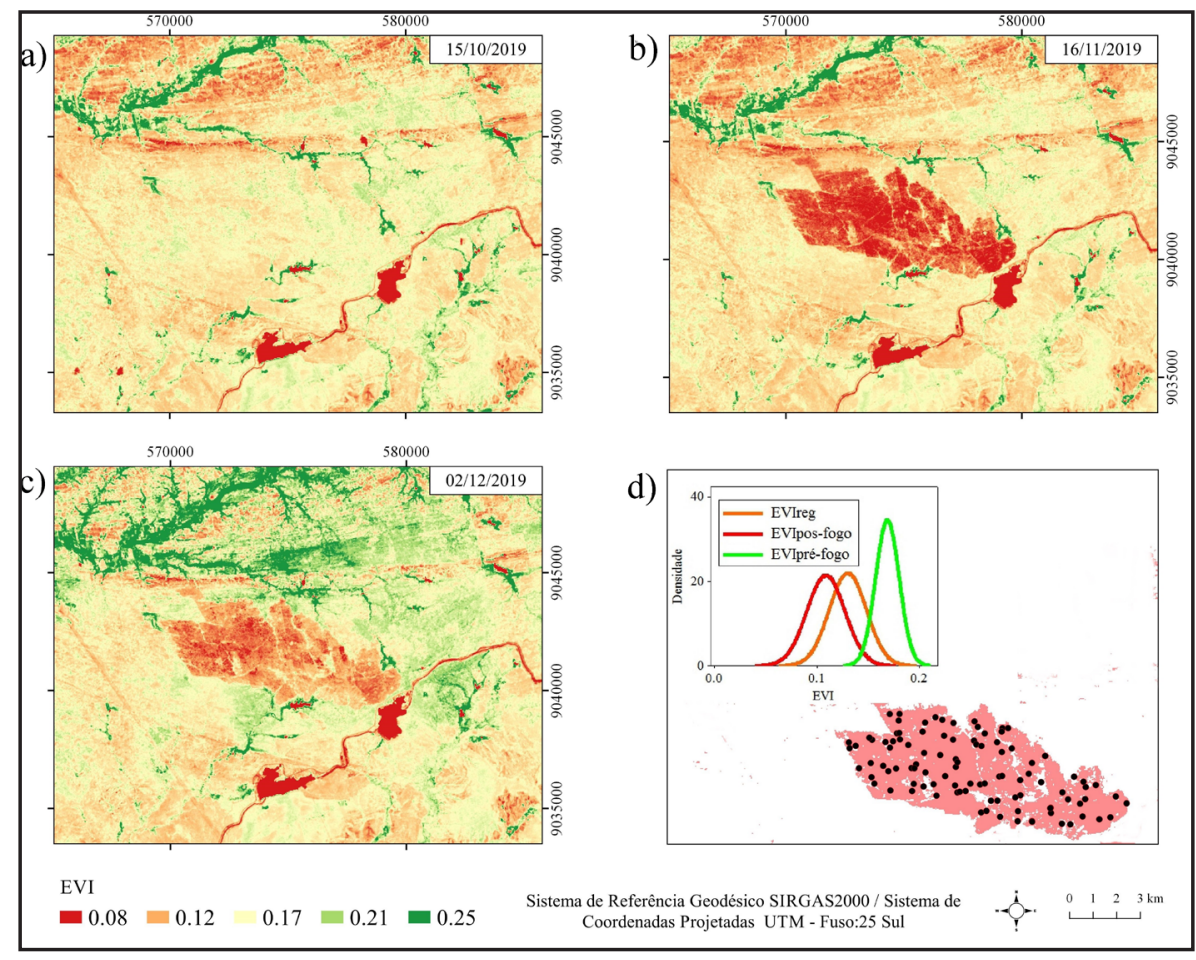

Fonte: Autores (2019)

A verificação da variação dos mapas de EVI permitiu dividir o comportamento 
da cobertura vegetal da área de estudo em três fases. A primeira apresenta-se por ausência de pixels de EVI relacionado ao incêndio, sendo os pixels negativos referentes ao corpo hídrico do reservatório e os canais da transposição do Rio São Francisco. Na segunda data foi possível observar pixels negativos na cobertura terrestre referente ao incêndio na área. No último mapa em análise, a vegetação dá sinais de regeneração com presença de pixels positivos próximos de zero.

Observou-se nas cartas imagens da Figura 2, que as áreas de incêndios e corpos hídricos aparecem com valores de EVI negativos. Esse comportamento está relacionado à alta absorção dessas feições nas faixas do vermelho, azul e na borda do infravermelho próximo, causando consequentemente uma diminuição de valores em índices espectrais nessas bandas. Em todo recorte há uma maior ocorrência de baixos valores de EVI $(>0,2)$, isso está associado ao deficit hídrico característico do bioma Caatinga. Essa evidência ocorre a partir do mês de julho até dezembro, o que torna a cobertura da vegetação seca, ocasionando o aumento da refletância do solo. Resultados semelhantes foram obtidos por outros autores. Formigoni, Xavier e Lima (2011), por exemplo, mostraram que nos diferentes biomas brasileiros, a Caatinga apresentou maior variação sazonal dos índices NDVI e EVI nos períodos de 2000 a 2006. Machado (2015), utilizando imagens MODIS, demonstrou que o EVI é capaz de detectar a diferença sazonal do vigor da vegetação entre os meses de estações seca e chuvosa, e entre a vegetação incendiada e não incendiada.

Os resultados do teste de normalidade são apresentados no gráfico da Figura 3, que indica uma boa concordância estatística sobre a normalidade da distribuição de dados de EVI para os três eventos em análise, mostrando, assim, maior probabilidade de os dados seguir uma distribuição gaussiana. Ou seja, a maioria dos dados do EVI ajustam-se a uma distribuição normal. Ressalta-se que a aquisição das amostras não foi obtida por acaso e que o conjunto amostral foi capaz de predizer todos os dados com nível de confiança de 95\%.

O teste de Kolmogorov-Smirnov confirma a hipótese de normalidade HO, pois a maioria dos pontos estão próximos à curva normal e os valores de $p$ obtidos foram maiores que o nível de significância (> 5\%). Além disso, para uma amostra de 100 
pixels, foi encontrado pela tabela de referência um valor crítico de 0,136 . Como os valores encontrados de $\mathrm{KS}<0,136$, não existe evidências para rejeitar a hipótese de normalidade dos dados. Portanto, o conjunto de amostras para os três eventos confirmam uma distribuição normal.

Figura 3 - Gráfico do Teste de Normalidade das amostras através do método de Kolmogorov-Smirnov

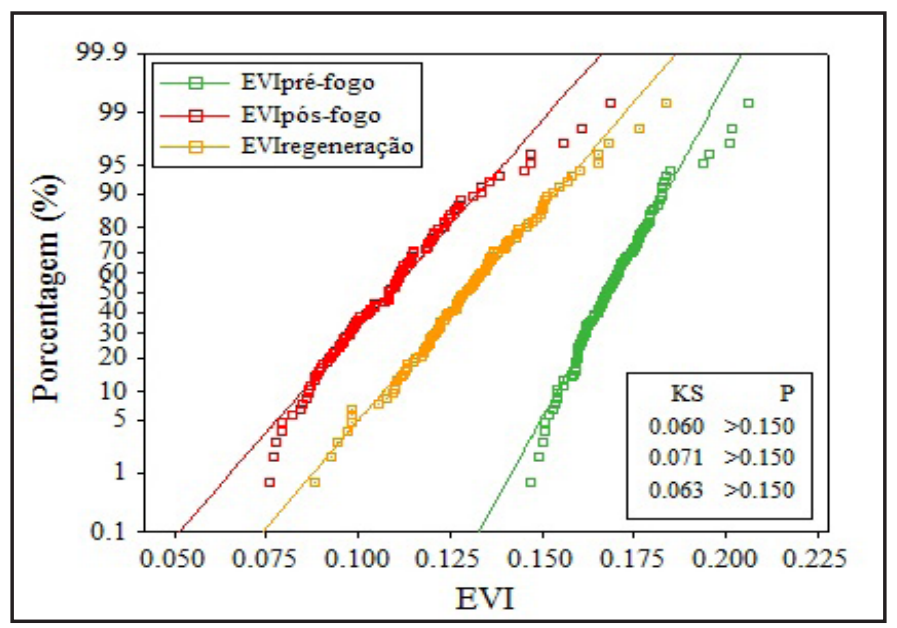

Fonte: Autores (2019)

A Figura 4 apresenta plotagem de amostras de pixels de EVI testadas para os três eventos: Pré-fogo, Pós-fogo e regeneração

Figura 4 - Gráfico boxplot das amostras de pixels de EVI testadas nos eventos de incêndio

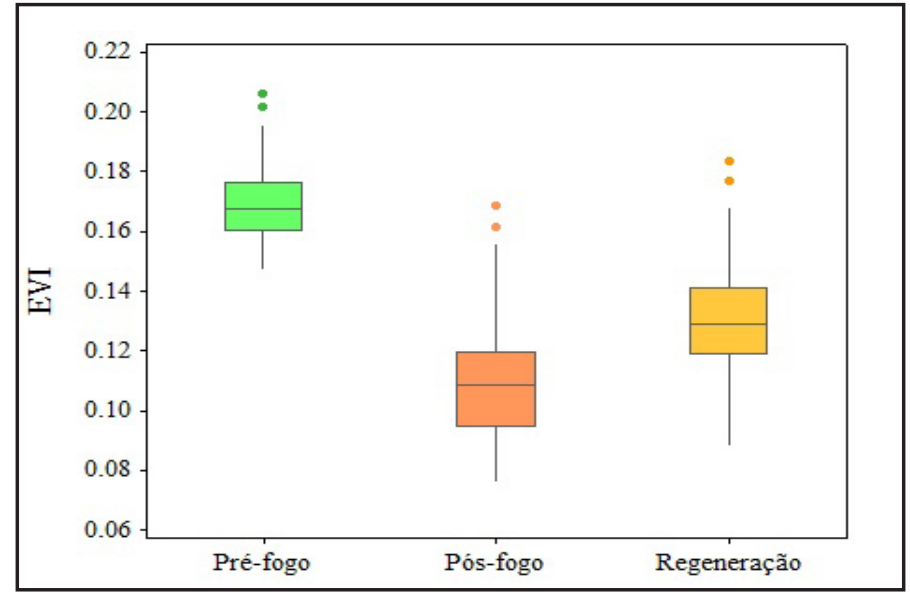

Fonte: Autores (2019) 
Observando-se a Figura 4 é possível verificar que antes do evento de incêndio as amostras de EVI apresentaram maior mediana e menor intervalo interquartil, evidenciando assim a homogeneidade de pixels de EVI, isto é, sem alterações importantes na cobertura vegetal. O EVIpós-fogo apresentou menor mediana da série provavelmente devido à perda da vegetação natural causada pelo incêndio. O EVIreg apresentou um aumento no valor da mediana, mostrando assim um bom desempenho da vegetação no processo de regeneração do bioma após aproximadamente 16 dias do incêndio. Apesar de todas as variáveis apresentarem outliers, apenas o EVIpós-fogo e EVIreg apresentaram desvio padrão e intervalo interquartil semelhantes, mostrando assim que os pixels estão com maior variabilidade nos valores de EVI.

A Figura 5 apresenta gráficos de dispersão das estimativas do EVIpós-fogoentre o EVIpré-fogoe o EVIreg. Essa análise de regressão pressupõe que o comportamento do EVIpós-fogo pode ser explicado a partir da dinâmica do EVIpré-fogo e EVIreg, gerando um modelo de regressão linear e atualização matemática analítico-preditiva de dados do EVI modificado pelo incêndio formado por um termo de intercepto (b) e um coeficiente angular (a). No processo de análise de dados de sensoriamento remoto através de modelagem preditiva por regressão linear, utilizam-se dados históricos. Esses dados viabilizam a estimativa de informações das alterações ocorridas na paisagem em períodos passados.

Em um desempenho ideal, espera-se que todos os pontos de amostra sejam plotados na linha de regressão e, consequentemente, a incompatibilidade entre a linha indicaria imperfeição do modelo. A Figura 5 mostra que o modelo em (a) e (b) apresentaram desempenho maior na avaliação da dispersão entre as amostras em relação ao EVIpós-fogo.

O teste de significância da regressão apresentada na Figura 5 indicou que o nível de confiança foi de 95\%, estatisticamente significativo $(P<0,05)$.

O EVIpós-fogoe o EVIreg apresentaram melhores ajustes ao modelo $\left(R^{2}=0,66\right)$ e estão fortemente correlacionados (Pearson $=0,81$ ), isso significa que as previsões 
geradas pelo modelo explicam aproximadamente $66 \%$ da variabilidade espaço temporal da regeneração da vegetação pós-fogo.

Figura 5 - Regressão linear entre a) EVIpós-fogo e EVIreg e c) EVIpós-fogo e EVIpré-fogo

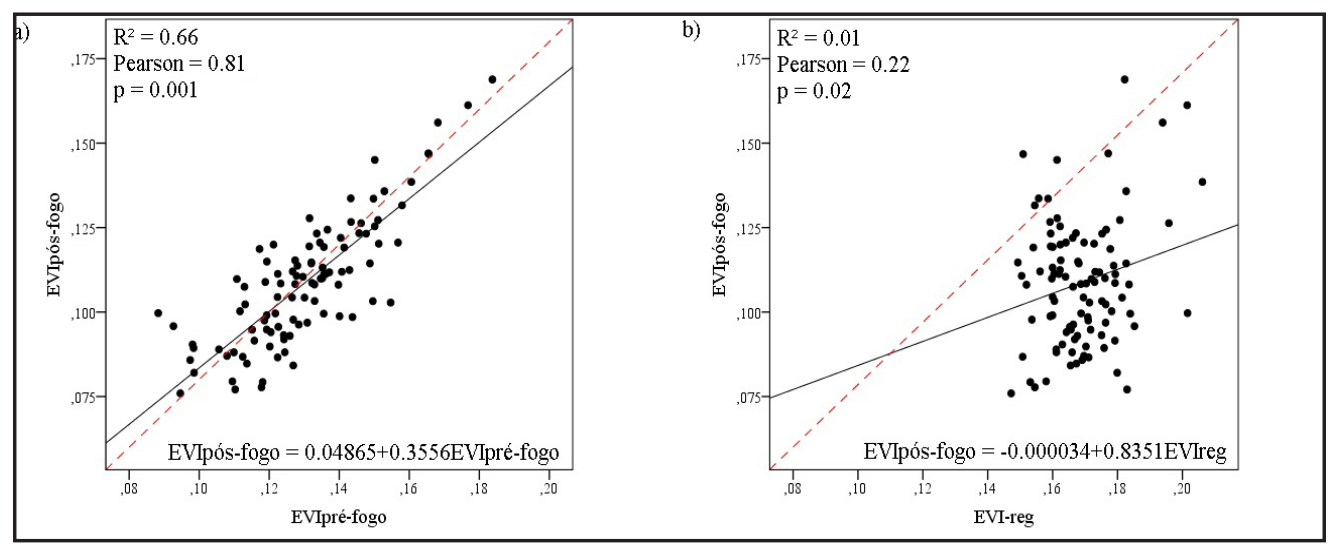

Fonte: Autores (2019

O EVIpré-fogoe o EVIpós-fogo apresentaram baixas relações $\left(R^{2}=0,01\right.$, Pearson $=0,22$ ), cujo modelo não foi capaz de gerar informações do incêndio a partir de dados de EVIpós-fogo. Consequentemente, o uso da linha de regressão obtida para os dados implicaria em uma forte generalização e inconsistências de informações. Isso também era esperado, uma vez que as áreas afetadas por incêndios levam a correlação menor com índices de vegetação no infravermelho próximo. Essa diferença pode estar relacionada à localização da região do incêndio, cujo clima quente e a forte sazonalidade no regime de chuvas resultam em tempos curtos para a recuperação da vegetação nativa.

Segundo Ponzoni e Shimabukuro (2010), as alterações na estrutura do mesofilo das folhas interferem no espelhamento interno da radiação incidente causando diminuição da reflectância de uma folha na região do infravermelho próximo. Os valores de correlação de Pearson para o índice EVI variam com a gravidade do incêndio, mostrando o mesmo comportamento do R². Entretanto, esse comportamento também permite afirmar que à medida que a gravidade do incêndio se enfraquecia, ou seja, presença de regeneração, o coeficiente de correlação também podia aumentar gradualmente em relação ao EVIpré-fogo. 
A Tabela 1 apresenta os autovalores e porcentagens de variâncias obtidos pela técnica de componentes principais.

Tabela 1 - Autovalores e proporções para as 3 primeiras Componentes Principais

\begin{tabular}{lcc}
\hline Componente Principal & Autovalores & Proporção (\%) \\
\hline CP1 & 1,88 & 62,8 \\
CP2 & 0,93 & 31,2 \\
CP3 & 0,18 & 6 \\
\hline
\end{tabular}

Fonte: Autores (2019)

Verificou-se na Tabela 1 que a primeira componente principal obteve autovalor acima de $1(\lambda>1)$ e foi responsável por $62,8 \%$ da variância no conjunto de dados. Seguindo o Critério de Kaiser (KAISER, 1958), apenas a primeira componente principal é significante para análise dos dados. A segunda componente principal foi também considerada pelo fato de os dados conterem outilers (mostrado no gráfico da Figura 4 de Boxplot) apresentando um autovalor de 0,93 (próximo a 1), que representa $31,2 \%$ da variância dos dados. Dessa forma, as duas primeiras componentes já explicam sozinhas 94\% da variância dos dados. Portanto, sua utilização possibilita a simplificação do número de variáveis, com perda de aproximadamente $6 \%$ do padrão de variabilidade amostral.

Diante disso, foram calculados os dois primeiros componentes principais e seus coeficientes de ponderação de cada característica (Figura 6). A seleção de dois componentes principais ocasionou a redução da dimensão de 3 variáveis originais para 2 componentes principais nas equações (8) e (9).

Figura 6 - Gráfico dos pesos de CP1 e CP2 para os EVIpré-fogo, EVIpós-fogo e EVIreg

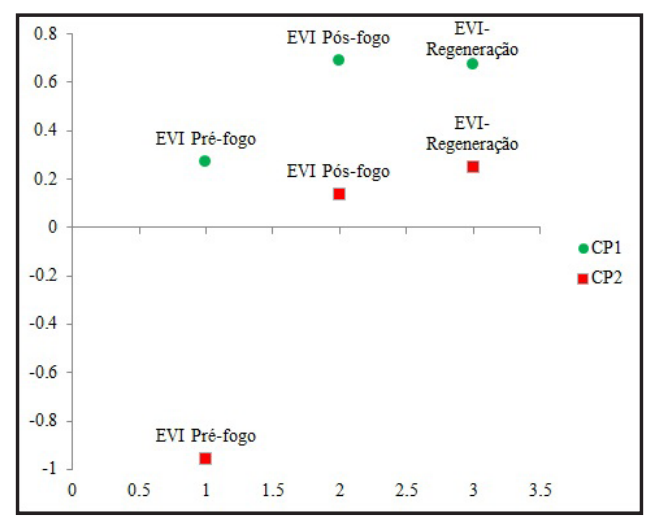

Fonte: Autores (2019) 
No gráfico de escores (Figura 7), pode ser observada a distribuição das amostras, as quais formaram três grupos, mostrando assim uma influência direta no comportamento dos dados referentes à espacialidade do local diante do evento pós-fogo e regeneração. Ao longo do eixo da CP1 positiva é possível observar a predominância de valores de escore EVIpós-fogo, destacando-se sua contribuição na variação na CP1. Além disso, o EVIpós-fogo está em maior número e mais distante da origem, o que retorna esta variável a causar um maior impacto no modelo. Sendo assim, o gráfico de escore corrobora a análise de proporção e autovetores.

O gráfico da Figura 7 também mostra que a CP2, no geral, está relacionada a valores de EVI com ausência ou baixa presença de incêndios. Com destaque para os valores negativos da CP2, associados à variável EVIpré-fogo, que por sua vez está inversamente correlacionada com a CP1, mostrando assim um comportamento de proporcionalidade inversa. Apresentando comportamento análogo à regressão linear, ou seja, quando o EVIpré-fogo aumenta e EVIpós-fogo diminui e vice-versa.

Figura 7 - Gráfico dos escores para CP1 e CP2 mostrando tendência de agrupamento das amostras analisadas

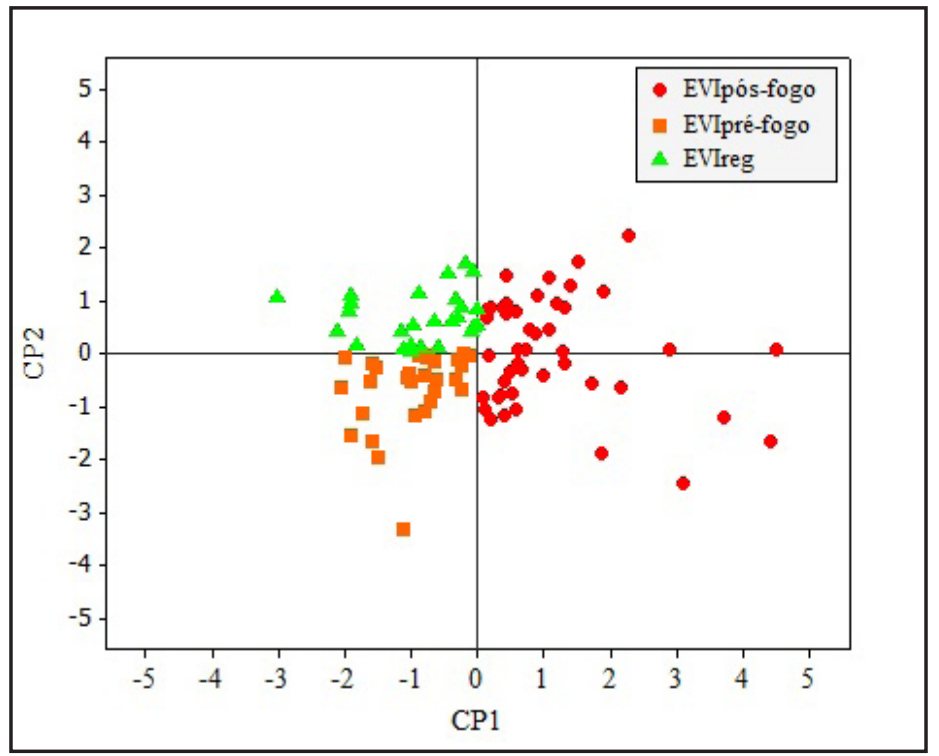

Fonte: Autores (2019) 
Na Figura 8, observou-se o gráfico de pesos e a relação entre as variáveis. Com base nessas relações foi possível inicialmente tentar inferir alguma interpretação geométrica para as componentes principais. Na Figura 1, é interessante notar a disposição das variáveis ao longo de CP1, que modela aproximadamente $62,8 \%$ da variância da matriz de dados. O EVIpós-fogo apresenta o maior peso, contribuindo mais para a CP1, refletindo a maior variabilidade dessa propriedade ao longo das variáveis. Assim, há razões para acreditar que a primeira componente principal (CP1) modela o comportamento dos valores do índice de vegetação EVI em eventos de incêndios.

Figura 8 - Gráfico Biplot da CP1 e CP2

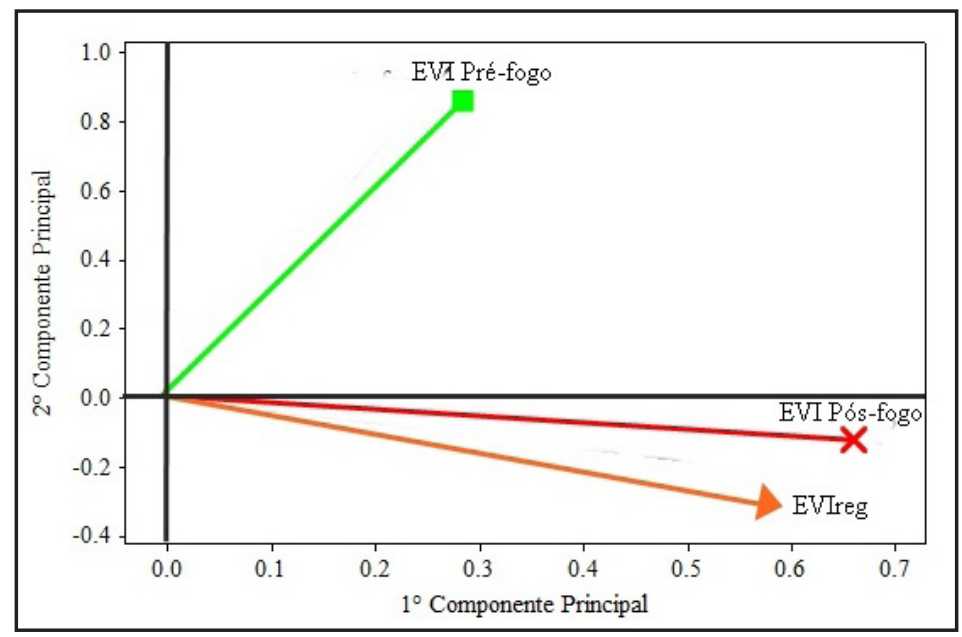

Fonte: Autores (2019)

A CP1 apresentou valores de coeficientes positivos com maiores contribuições para as variáveis EVIpós-fogo e EVIreg. A CP2 apresentou baixos valores de coeficientes. É importante destacar o coeficiente negativo para a variável EVIpré-fogo. Dessa forma, a CP1 pode ser chamada de componente de áreas afetadas pelo incêndio,uma vez que as variáveis EVIpós-fogo e EVIreg se destacam neste componente de acordo com os coeficientes de ponderação e também de correlação contidos na Tabela 2. A CP2, pelo contraste presente entre a variável EVIpré-fogo observada na equação e na Tabela 2, pode ser chamada de componente de áreas não incendiadas. 
A matriz de autovetor, apresentada nas Equações (8) e (9), mostrou valores bastante próximos nos coeficientes das variáveis EVIreg e EVIpós-fogo. Caracterizando que essas são variáveis que mais se relacionaram com a primeira componente principal. A primeira componente principal está correlacionada positivamente com os índices EVI. Portanto, qualquer variabilidade nos pixels de EVIpós-fogo é proporcional ao valor da primeira componente principal.

$$
\begin{aligned}
& C P 1=0.27 X 1+0.68 \times 2+0.67 X 3 \\
& C P 2=-0.95 X 1+0.13 \times 2+0.24 X 3
\end{aligned}
$$

A segunda componente principal mostra o contraste nos sinais no EVIpré-fogo e baixos valores dos coeficientes EVIpós-fogoe EVIreg, assim, este componente mede principalmente a variabilidade do deslocamento da variável EVIpré-fogo antes do incêndio ao longo da CP1. Demostrando a variação dos dados de forma decrescente e independente. A Figura 8 também mostra a disparidade das equações no plano. Dessa forma, a multiplicidade dimensional dos dados é condensada a um espaço bidimensional, garantindo máxima mudança e reforçando as diferenças nas séries temporais. A Figura 8 mostra a disposição dos eixos das variáveis EVIpré-fogo, EVIpósfogo e EVIreg, o espaço bidimensional da CP1 e CP2 num gráfico biplot.

As variáveis EVIpós-fogo e EVIreg apresentam maiores contribuições para a CP1, isso é observado no gráfico biplot na Figura 8, na qual essas variáveis possuem os vetores de maior comprimento e mais próximos do eixo da CP1. O EVIpré-fogo além de ser de menor comprimento está próximo do eixo da CP2, portanto sua contribuição não é tão similar em relação às variáveis EVIpós-fogo e EVIreg. Observa-se que as disposições dos eixos são coerentes com as Equações (8) e (9), nas quais as duas variáveis de maior contribuição têm maior peso na CP1 (Equação 8). Enquanto o outro eixo perpendicular tem maior peso de representação negativa na CP2. Nesse caso, interpreta-se que a informação da área alterada por incêndio se comporta através de eixo da CP1. 
O comportamento simultâneo, das componentes principais na análise dos três eventos (pré-fogo, pós-fogo e regeneração), mostrou que o impacto de áreas atingidas por incêndios obteve variações importantes acerca do dossel vegetativo, de forma rápida, não destrutiva e em tempo real, contribuindo para tomada de decisão quanto ao manejo de áreas próximas à barragem geradora de energia.

Observou-se que a natureza parece altamente correlacionada com os dados de satélite, pois eles produziram um resultado semelhante quando comparado ao modelo ACP e Regressão Linear. Ambos os casos resultaram em baixas correlações para os eventos pré e pós-fogo. Além disso, permitiu estimar a dinâmica da cobertura vegetal em uma área de clima singular e com baixas frequências de incêndios de grande porte.

Os resultados deste estudo corroboraram as afirmações de Wallace, Webb e Thomas (2008), em que modelo baseado em EVI permitiu uma melhor interpretação direta e correlação com a cobertura vegetal, enquanto a inspeção por componentes principais foi no geral mais eficiente. Evidentemente por se tratar de correlações estatísticas com vários agrupamentos de dados independentes e sem qualquer perda de informação. Alexandris, Koutsias e Gupta (2017) mostraram, através de dados temporais Landsat 5 TM e MODIS, que a primeira e a segunda componente principal possuem maior sinal na área afetada pelo fogo, e as componentes acima de 3,4,5 e 6 apresentaram bastante ruído e baixo sinal, portanto podem ser seguramente descartadas. Contudo, os dados transformados por componentes principais e a análise por regressão linear simples foram superiores aos dados originais do sensor de satélite no plano de índices de vegetação. A criação de um novo espaço espectral permitiu o maior aprimoramento quantitativo e de sinal espectral da vegetação, que sofreu incêndios, bem como a sua regeneração (KOUTSIAS; MALLINIS; KARTERIS, 2009). 


\section{CONCLUSÕES}

A quantificação das características da Caatinga derivadas do índice espectral EVI de imagens Landsat-8/OLI proporcionou uma melhor avaliação de suas condições biofísicas sob efeitos do fogo.

O teste de normalidade Kolmogorov-Smirnov foi suficiente para comprovar que os dados seguem uma distribuição normal, com um nível de significância de 95\%. Os métodos estatísticos de Regressão Linear e Análise por Componentes Principais possibilitaram diagnósticos importantes nas estimativas e/ou relacionamentos entre as variáveis aleatórias.

A Regressão Linear foi capaz de explicar os parâmetros de determinação entre os índices de EVl, corroborando a redução de dimensionamento dos dados obtidos pela técnica de Análise por Componentes Principais. As componentes principais CP1 e a CP2 apresentaram resultados com 94\% da variância dos dados. Fornecendo, dessa forma, indicativos importantes na tomada de decisões sobre o manejo de áreas de incêndios próximas à barragem geradora de energia.

Constatou-se neste estudo que nem sempre é viável seu uso com dados de campo, principalmente quando se trata de áreas extensas e dados históricos em eventos de fogo de frequências não usuais. Nesse caso, foram compilados os dados existentes, e foi possível mostrar a caracterização da complexidade da paisagem em eventos de incêndios próximos ao reservatório de Itaparica, que possui grande importância energética para o Brasil.

A metodologia apresentada neste estudo demonstrou ser uma alternativa relevante na estimativa quantitativa da vegetação de Caatinga afetada por eventos, que normalmente não são capturadas pelos mapas tradicionais existentes na área. Os mapas resultantes representam um aprimoramento importante no mapeamento da distribuição da vegetação. Este estudo gera indicativos para futuras pesquisas científicas vinculadas ao gerenciamento do espaço relacionado à vulnerabilidade e recuperação de paisagens de vegetação do clima semiárido sob situações de incêndios. 


\section{REFERÊNCIAS}

ABOELGHAR, M. et al. Retrieving leaf area index from SPOT4 satellite data. The Egyptian Journal of Remote Sensing and Space Science, [s. I.], v. 13, n. 2, p. 121-127, 2010.

ALEXANDRIS, N.; KOUTSIAS, N.; GUPTA, S. Remote sensing of burned areas via PCA, Part 2: SVDbased PCA using MODIS and Landsat data. Open Geospatial Data, Software and Standards, [s. I.], v. 2, n. 21, 2017. DOI: https://doi.org/10.1186/s40965-017-0029-0

BOWMAN, D. M. et al. Fire in the earth system. Science, New York, v. 324, p. 481-484, 2009.

CAMPOS, S. et al. Closure and partitioning of the energy balance in a preserved area of a Brazilian seasonally dry tropical forest. Agricultural and Forest Meteorology, Amsterdam, v. 271, p. 398-412, 2019.

CHAVEZ JUNIOR, P. S. An improved dark-object subtraction technique for atmospheric scattering correction of multispectral data. Remote Sensing of Environment, New York, v. 24, p. 459-479, 1988.

CHUVIECO, E. Teledeteccion Ambiental. La observacion de la Tierra desde el espacio. Barcelona: Editorial Ariel, 2010. 528 p.

COMPANHIA DE PESQUISA DE RECURSOS MINERAIS (Brasil). Serviço Geológico do Brasil. Projeto cadastro de fontes de abastecimento por água subterrânea. Diagnóstico do município de Floresta, Estado de Pernambuco. [S. I.], 2005. 33 p.

CORDER, G. W.; FOREMAN, D I. Nonparametric statistics for non-statisticians: a step-bystep approach.[S. I.]:John Wiley \& Sons, 2011. 264 p.

DENG, J. et al. A methodology to monitor urban expansion and green space change using a time series ofmulti-sensor spot and sentinel-2a images. Remote Sensing, Basel, v. 11, n. 10, p. 1-21, 2019.

FERNANDES, P. M. et al. Bottom-up variables govern large-fire size in Portugal. Ecosystems, New York, v. 19, p. 1362-1375, 2016.

FORMIGONI, M. H.; XAVIER, A. C.; LIMA, J. S. S. Análise Temporal da Vegetação na Região do Nordeste através de dados EVI do MODIS. Ciência Florestal, Santa Maria, v. 21, n. 1, p. 1-8, 2011.

GAITANI, N. et al. High-resolution spectral mapping of urban thermal properties with unmanned aerial vehicles. Building Environment, Oxford, v. 121, p. 215-224, 2017.

GALVÃO, L. S. et al. On intra-annual EVI variability in the dry season of tropical forest: a case study with MODIS and hyperspectral data. Remote Sensing of Environment, New York, v. 115, n. 9, p. 2350-2359, 2011.

GAO, X. et al. Optical-biophysical relationships of vegetation spectra without background contamination. Remote Sensing of Environment, New York, v. 74, n. 3, p. 609-620, 2000. 
HUETE, A. R. et al. Amazon rainforests green up with sunlight in dry season. Geophysical Research Letters, Medford, v. 33, n. L06405, 2006.

HUETE, A. R. et al. A comparison of vegetation indices over a global set of TM images for EOSMODIS. Remote Sensing of Environment, New York, v. 59, n. 3, p. 440-451, 1997.

IBGE. Metadados2016. [S. I.], 2016. Disponível em: ftp://geoftp.ibge.gov.br. Acesso em: 21 jan. 2020.

INTERGOVERNMENTAL PANEL ON CLIMATE CHANGE. Climate Change 2013: The Physical Science Basis. Contribution of Working Group I to the Fifth Assessment Report of the Intergovernmental Panel on Climate Change. Cambridge: Cambridge University Press, 2013. $1535 \mathrm{p}$.

ISLAM, T. U. Ranking of normality tests: an appraisal through skewed alternative space. Symmetry, [s. I.], v. 11, n. 7, p. 1-14, 2019.

KAISER, H. F. The varimax criterion for analytic rotation in fator analysis. Psychometrika, New York, v. 23, n. 3, p. 187- 200, 1958.

KOUTSIAS, N.; MALLINIS, G.; KARTERIS, M. A forward/backward principal component analysis of Landsat-7 ETM+ data to enhance the spectral signal of burnt surfaces. ISPRS Journal of Photogrammetry and Remote Sensing, Amsterdam, v. 64, n. 1, p. 37-46. DOI: doi:10.1016/j. isprsjprs. 2008.06.004

LIZUNDIA-LOIOLA, J.; PETTINARI, M. L.; CHUVIECO, E. Temporal Anomalies in Burned Area Trends: Satellite Estimations of the Amazonian 2019 Fire Crisis. Remote Sensing, Basel, v. 12, n. 151, p. 1-8, 2020.

MACHADO, G. C. Efeitos dos incêndios florestais na vegetação do cerrado utilizando dados do sensor MODIS. 2015. Dissertação (Mestrado em Engenharia Florestal) - Universidade de Brasília, Departamento de Engenharia Florestal, Brasília, 2015.

MARIANO, D. A. et al. Use of remote sensing indicators to assess effects of drought and human induced land degradation on ecosystem health in Northeastern Brazil. Remote Sensing of Environment, New York, v. 213, p. 129-143, 2018.

MISHRA, P. et al. Descriptive statistics and normality tests for statistical data. Annals of Cardiac Anaesthesia, [s. I.], v. 22, n. 1, p. 67-72, 2019.

MOLINA, D. M. Origins of Arson in Northwestern Spain. Fire Management Notes, Washington, v. 57, n. 3, p. 18-23, 1997.

PONZONI, F. J.; SHIMABUKURO, Y. E. Sensoriamento remoto no estudo da vegetação. São José dos Campos: Parêntese, 2010. 127 p.

THENKABAIL, P. S.; LYON, J. G.; HUETE, A. Hyper spectral remote sensing of vegetation. Boca Raton: CRC Press, 2016. 782 p.

UNITED STATES GEOLOGICAL SURVEY. Heart Explorer Data System. Base de dados 2019. USA, 2019. Disponível em: https://earthexplorer.usgs.gov/. Acesso em: 22 nov. 2019.

WALLACE, C. S. A.; WEBB, R. H.; THOMAS, K. A. Estimated distribution of perennial vegetation cover in the Mojave desert using MODIS-EVI data. GI Science and Remote Sensing, USA, V. 45, n. 2, p. 167-187, 2008. 


\section{Contribuição de Autoria}

\section{1 - Juarez Antônio da Silva Junior}

Engenheiro de Cartografia e de Agrimensura

https://orcid.org/0000-0002-2898-0309•juarez.silvajunior@ufpe.br

Contribuição: Conceituação, Curadoria de dados, Análise Formal, Obtenção de financiamento, Investigação, Metodologia, Administração do projeto, Recursos, Software, Supervisão, Validação, Visualização, Escrita - primeira redação, Escrita revisão e edição

\section{2 - Admilson da Penha Pacheco}

Físico, Dr., Professor

https://orcid.org/0000-0002-3635-827X•pacheco3p@gmail.com

Contribuição: Conceituação, Curadoria de dados, Análise Formal, Obtenção de financiamento, Investigação, Metodologia, Administração do projeto, Recursos, Software, Supervisão, Validação, Visualização, Escrita - primeira redação, Escrita revisão e edição

\section{Como citar este artigo}

Silva Junior, J. A.; Pacheco, A. P. Avaliação de incêndio em ambiente de Caatinga a partir de imagens Landsat-8, índice de vegetação realçado e análise por componentes principais. Ciência Florestal, Santa Maria, v. 31, n. 1, p. 417-439, 2021. DOI 10.5902/1980509843818. Disponível em: https://doi.org/10.5902/1980509843818. Acesso em: xx mês abreviado 202x. 